\title{
Identificação das Funcionalidades de um Assistente de Comunicação aos Surdos Aplicando Técnicas de Elicitação de Requisitos
}

\author{
Gabriel Araújo Belém \\ Instituto Federal do Amazonas - IFAM \\ Manaus, AM, Brasil \\ 2020001958@ifam.edu.br \\ Priscila Silva Fernandes \\ Instituto Federal do Amazonas - IFAM \\ Manaus, AM, Brasil \\ priscila.fernandes@ifam.edu.br
}

\author{
Gabriela Fidelis de Oliveira \\ Instituto Federal do Amazonas - IFAM \\ Manaus, AM, Brasil \\ 2020008420@ifam.edu.br
}

\author{
Vitor Bremgartner da Frota \\ Instituto Federal do Amazonas - IFAM \\ Manaus, AM, Brasil \\ vitorbref@ifam.edu.br
}

\author{
Luiz Sousa da Silveira \\ Instituto Federal do Amazonas - IFAM \\ Manaus, AM, Brasil \\ 2020008609@ifam.edu.br \\ Luis Rivero \\ PPGCC/UFMA \\ São Luis, MA, Brasil \\ luis.rivero@ufma.br
}

\begin{abstract}
Deaf people have difficulty communicating due to the lack of mastery of Libras (Brazilian sign language for deaf citizens). New systems have emerged to support the communication of people with hearing disabilities with regard to: simultaneous translation, teaching of Libras and presentation of animations. However, these systems do not have ready-made phrases or support for emergency situations. Considering this scenario, this article presents the proposal of the Communication Assistant to the Deaf (AComS), which aims to support the communication of people with hearing loss, complementing the features already presented by existing systems such as VLibras, HandTalk and MIDOAA. After the application of requirements gathering techniques such as document analysis and benchmarking, a high-fidelity prototype of the system was proposed, which presents the various features of AComS in its mobile version.
\end{abstract}

\section{PALAVRAS-CHAVE}

Engenharia de Software, Inclusão Digital, Interação Humano-Computado

\section{INTRODUÇÃO}

Pessoas surdas têm dificuldades no meio social em acessar serviços e se comunicar encontrando muitas barreiras como acessibilidade e acesso à informação. Devido a isso, surgiu o decreto de número 5.626 [1] no qual destaca-se o resgate da educação como um bem social, garantindo condições para enfrentar as dificuldades da sociedade contemporânea. Onde são concedidos direitos por meio da inclusão, garantia no atendimento às necessidades especiais e implementação de novos mecanismos que visam permitir, viabilizar e facilitar a interação nos meios alternativos de comunicação dos deficientes auditivos.

Apesar da normativa de implantação de serviços de atendimento para auxiliar na comunicação de pessoas com deficiência auditiva, ainda existem situações em que uma pessoa surda não pode se comunicar facilmente. A partir dessas novas expectativas, a comunicação limitada dá lugar a comunicação auxiliada, muito apreciada pelos surdos.
Nesse contexto, softwares especializados podem surgir para auxiliar na comunicação entre deficientes auditivos e ouvintes, resguardando os propósitos de ensino, comunicação em atendimentos médicos e pesquisa. Considerando a importância de se desenvolver sistemas de qualidade para apoiar na comunicação de pessoas com deficiência auditiva, neste artigo é apresentada a proposta do Assistente de Comunicação ao Surdo (AComS).

Para definir as funcionalidades do aplicativo, foi aplicada a metodologia Scrum, com a ajuda das técnicas de benchmarking e análise de documentos, com o intuito de reduzir os riscos do projeto e maximizar o valor do produto elaborado para atingir a funcionalidade a aproveitamento desejado. Deste modo, tem-se como meta utilizar a tecnologia como recurso a fim de atender e facilitar a comunicação entre surdos e não surdos, propiciando uma socialização inovadora e inclusiva.

\section{SOLUÇÃO PROPOSTA}

\subsection{Metodologia}

Utilizando uma metodologia baseada em Scrum para analisar especificações e levantar os requisitos para propor a interface gráfica do sistema, foram utilizadas as técnicas de benchmarking e análise de documentos, ferramentas fundamentais e amplamente criteriosas no desenvolvimento de sistemas computacionais e na construção de softwares, considerando também que aplicativos móveis são úteis e eficientes na potencialização do ensino, da aprendizagem, da comunicação e inclusão social da pessoa surda.

Na técnica baseada em benchmarking, compararam-se as funcionalidades dos concorrentes existentes. Aplicando a técnica de análise de documentos, foi possível observar um vasto conhecimento no que tange o cenário de aplicações móveis para surdos, sujeitos com capacidade e necessidades diferenciadas, público desta pesquisa, comparando-se os artigos científicos e estudos sobre os sistemas concorrentes [2-6]. Assim, levantando requisitos significativos na Tabela 1.

\subsection{O Sistema AComS}

A criação de uma comunicação entre deficientes auditivos e ouvintes, por meio de um software emissor de sons, sinais, imagens e 
XII Computer on the Beach

07 a 09 de Abril de 2021, Online, SC, Brasil

Belém et al.

Tabela 1: Identificação de requisitos

\begin{tabular}{l|l}
\hline Requisitos & Descrição \\
\hline RF001 & $\begin{array}{l}\text { O sistema deve oferecer “botão do pânico" para } \\
\text { emergências. }\end{array}$ \\
\hline RF002 & $\begin{array}{l}\text { O sistema deve fazer ligações por vídeo chamada } \\
\text { usando algum software compatível. }\end{array}$ \\
\hline RNF001 & $\begin{array}{l}\text { O sistema deve ter tradução de pelo menos 2 idio- } \\
\text { mas para LIBRAS. }\end{array}$ \\
\hline RI001 & O sistema não pode ser de difícil manuseio. \\
\hline
\end{tabular}

textos para o usuário, assume grande importância devido a possibilidade de maior interatividade no meio social, amenizando processos de segregação social. Os concorrentes identificados V LIBRAS, Hand Talk e MIDOAA oferecem ferramentas de tradução simultânea, aplicação de animações, captação de sons e manuseamento em todos os lugares. Entretanto, na análise de benchmarking, foram identificadas as necessidades de: atualizações periódicas do sistema, fácil manuseio e uma resposta rápida a situações de perigo para o deficiente, propondo frases prontas para uma ocasião de socorro, por meio de reprodução de áudio das mesmas, ou seja, um botão do pânico. Além disso, foi pensada uma função de chamada de vídeo para um contato previamente cadastrado pelo usuário.

Como proposta inicial, foi desenvolvido um protótipo de alta fidelidade na ferramenta Microsoft PowerPoint. As Figuras 1 e 2 apresentam partes do protótipo desenvolvido.

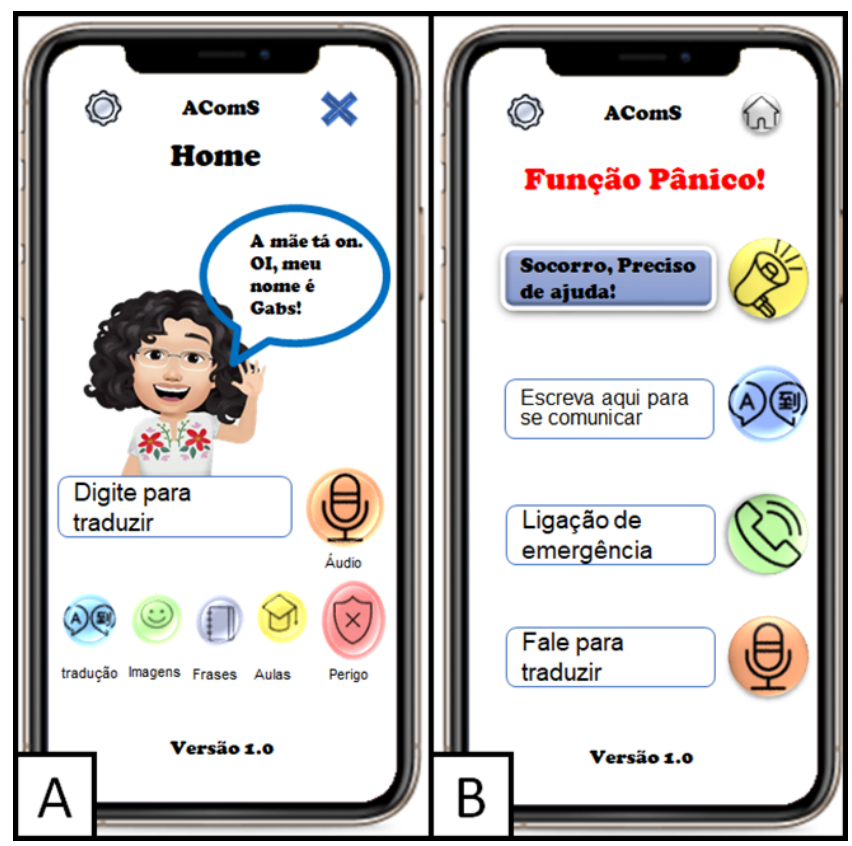

Figura 1: Protótipo de Interface: (A) Tela Inicial do Protótipo e (B) Interface do botão do pânico.

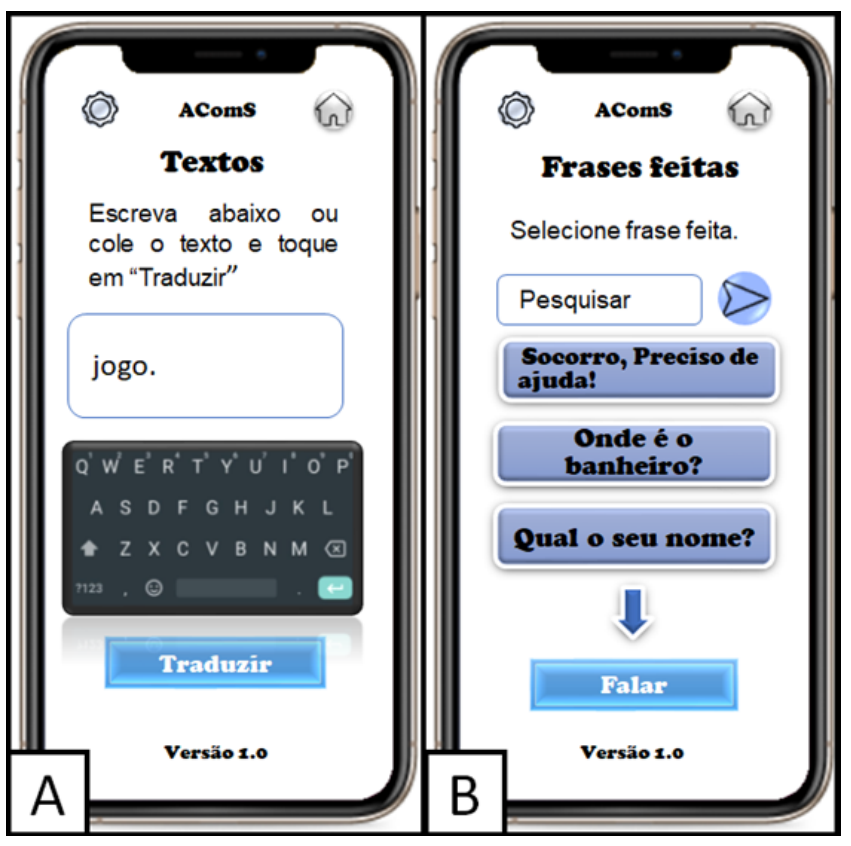

Figura 2: Protótipo de Interface: (A) Tradução Simultânea de Texto para Libras e (B) Funcionalidade de Ensino de Frases Feitas.

\section{CONCLUSÕES}

A utilização de novas tecnologias facilitadoras da inclusão social e comunicação e os aplicativos de um modo geral promovem a interação e a socialização de surdos e ouvintes, bem como de ouvintes e surdos, minimizando desigualdades e processos de baixa estima, uma vez que os processos de socialização - elemento fundamental da constituição humana, estão inerentemente associados aos de interação.

Além da aplicabilidade do software no processo citado, medidas educacionais em contextos distintos, podem se valer da positividade do aplicativo como promoção da inclusão social, digital e instrumento motivacional com as devidas adequações nos processos e abordagens escolhidas, pois fortalecem o potencial inclusivo e acessibilidade.

Em se tratando de um protótipo, outros estudos e pesquisas de novas aplicações, estão em desenvolvimento para que os procedimentos estimulem e possibilitem novas experiências com aplicativos para surdos. Há que se definir que a criação do software deve atender a intencionalidade da inclusão, onde a fácil comunicação e o desenvolvimento socioemocional sejam assegurados pela especificidade do aplicativo, uma vez que propicia grandes benefícios de conectividade, ampliação do arcabouço linguístico e da necessidade do usuário, com a socialização e autonomia na utilização, no acesso e redução nas limitações.

\section{AGRADECIMENTOS}

Este trabalho foi realizado com o apoio do Projeto Aranouá, convênio realizado entre o Instituto Federal do Amazonas (IFAM) e a Samsung Eletrônica da Amazônia (SEDA). 


\section{REFERÊNCIAS}

[1] República Federativa do Brasil Casa Civil Subchefia para Assuntos Jurídicos. Decreto $\mathrm{n}^{\circ} 5.626$, de 22 de dezembro de 2005. 2005.

[2] Igor Farias De Oliveira, João Lucas Torres De Sousa, Larissa Cabral de Carvalho, Maria Durciane Oliveira Brito, Kátia Maria de Aguiar Freire, and Ana Christina de Sousa Damasceno. A utilização do aplicativo vlibras como forma de ensino e aprendizagem para alunos surdos. Revista Psicologia \& Saberes, 9(16):22-30, 2020

[3] Francisco Joilsom Carvalho SARAIVA, Reinaldo dos Santos MOURA, and Rose Fabiana de Medeiros dos Santos. A voz das mãos: o uso do aplicativo hand talk na consulta de pré-natal com uma gestante surda. Encontro Alagoano de Educação
Inclusiva, 1(1), 2015.

[4] Andreza Mourão, Christian Menezes, Arcanjo Lopes, and José Francisco Netto. App midoaa: Objeto de aprendizagem acessível para apoiar estudantes com deficiência auditiva. In Anais dos Workshops do Congresso Brasileiro de Informática na Educação, volume 8, page 1140, 2019.

[5] Luana Silva Reis, Tiago Maritan U de Araújo, Maria Dayane F Cirino Lima, Angelina S da Silva Sales, and Yuska Paola Costa Aguiar. Avaliação de usabilidade do aplicativo vlibras-móvel com usuários surdos. In Anais Estendidos do XXIII Simpósio Brasileiro de Sistemas Multimídia e Web, pages 123-126. SBC, 2017.

[6] Selma Regina Paixão. Hand talk: Aplicativo móvel para auxiliar na comunicação com os surdos. 